\title{
¿Es momento de adoptar el DAPSA para la evaluación de la Artritis Psoriásica?
}

\author{
Enrique R. Soriano \\ Sección Reumatología, Servicio de Clínica Médica. Hospital Italiano de Buenos Aires.
}

La mejor forma de evaluar la respuesta al tratamiento en artritis psoriásica no está aún definida ${ }^{1}$. Importantes esfuerzos están siendo realizados por grupos como GRAPPA (Group for Research and Assessment of Psoriasis and Psoriatic Arthritis) y OMERACT (Outcome Measures in Rheumatology), para validar instrumentos para la medición de la actividad de esta enfermedad y para encontrar algún instrumento que evalúe la misma en forma global2,3. Encontrar un solo instrumento que sea capaz de evaluar la enfermedad en sus principales y distintos dominios de compromiso (piel, articulaciones, compromiso axial, uñas, entesitis y dactilitis), es difícil, por la desconexión que muchas veces existe entre la actividad de los mismos ${ }^{4,5}$. Sin embargo, este esfuerzo tiene sentido si consideramos que es una enfermedad con múltiples manifestaciones, y para facilitar el acceso a drogas más efectivas, ya que por ejemplo pacientes que podrían no cumplir criterios para recibir un tratamiento biológico para cada compromiso en forma individual, debieran recibirlo si se consideran todos los compromisos en forma conjunta.

En este número de la revista, Gallino y cols. presentan un prolijo y elegante trabajo de validación y presentación de valores de corte para distintos estados del DAPSA, score que evalúa solo el compromiso articular periférico ${ }^{6}$. En ese sentido es muy similar al DAS28, excepto que contempla la evaluación de 66/68 articulaciones. Este punto que no es menor al momento de la evaluación individual, ya que por ejemplo alrededor de un 30\% de pacientes no ingresarían a un ensayo clínico de artritis psoriásica si se consideran solo 28 articulaciones, lo es mucho menos al momento de realizar estudios poblacionales, como queda reflejado por la alta correlación entre ambos índices. El estudio de Gallino y cols. está bien diseñado y ejecutado, aunque algunos puntos merecen ser comentados. En primer lugar, los valores de corte fueron establecidos comparando con valores de corte del SDAI. Teniendo en cuenta que ambos puntajes están compuestos prácticamente por las mismas variables, es esperable su alta correlación, y el excelente funcionamiento y discriminación de los mismos. Sin embargo, no se pudieron encontrar valores de corte que funcionaran adecuadamente cuando se utilizó la opinión de los expertos, y esto es un llamado de atención. En segundo lugar, no está detallado cómo se eligieron los valores mínimo y máximo de cada categoría, y esto es importante porque estos valores suelen superponerse y en general su elección final termina siendo una decisión arbitraria. El verdadero valor de puntos de corte, de un score o puntaje compuesto es establecido cuando se demuestra menor progresión radiológica y mejor calidad de vida en el mediano y largo plazo, de los pacientes que logran el estado de remisión o baja actividad, comparado con los pacientes que se mantienen en mediana o alta actividad. Esto ha sido recientemente demostrado por Aletaha y Smolen utilizando las bases de datos de los ensayos clínicos de Infliximab (IMPACT II) y de golimumab (Go-Reveal) ${ }^{7}$. Esto mismo también está demostrado para MDA (Minimal Disease Activity), una herramienta de evaluación de respuesta de la artritis psoriásica, que contempla además de las articulaciones periféricas, la piel y la entesitis ${ }^{89}$.

El MDA es un simple y excelente método para evaluar el estado de remisión, o mínima actividad, que ha demostrado no solo validez, sino ser adecuado para la toma de decisiones, y utilización en un esquema de tratamiento por objetivos (Treat To Target) ${ }^{10}$.

La remisión clínica puede ser conceptualizada como un estado en el que hay completa ausencia de actividad de la enfermedad, sin signos ni síntomas de enfermedad activa ${ }^{1,11}$, y esta definición requiere ausencia de actividad en 
todas las facetas de la misma.

Es por esta razón que se sigue aún en la intensa búsqueda de índices que tomen esta conceptualización en consideración.

El trabajo de Gallino y cols., al validar el índice DAPSA, y presentar valores de corte, nos ayuda a avanzar en la búsqueda de la mejor forma de evaluar a nuestros pacientes con artritis psoriásica. Sin embargo, no deja de ser un índice más, dentro de los múltiples disponibles, que solo tiene en consideración el compromiso articular periférico, y no define un estado de remisión completo de la enfermedad. Por lo tanto, para responder a la pregunta del título del editorial, no, personalmente no creo que sea el momento de adoptar el DAPSA para la evaluación de nuestros pacientes con artritis psoriásica.

Debemos seguir en la búsqueda de una definición de remisión o baja actividad que abarque la enfermedad en forma completa, que sea práctico y fácil de utilizar en la clínica diaria, adecuado para los ensayos clínicos, aceptado por los financiadores y entes reguladores, y se relacione con la evolución a largo plazo de los pacientes. A mi entender, aun con sus imperfecciones, el que mejor cumple con estos criterios, hasta el momento, es el MDA, definido por Coates y colaboradores ${ }^{8}$ hace algunos años.

\section{Bibliografía}

1. Soriano ER. Defining remission in psoriatic arthritis: are we getting closer? The Journal of rheumatology 2015;42(6):907-8. doi: 10.3899/jrheum.150331

2. Coates LC, Fitzgerald O, Mease PJ, et al. Development of a Disease Activity and Responder Index for Psoriatic Arthritis -- Report of the Psoriatic Arthritis Module at OMERACT 11. The Journal of rheumatology 2014 doi: 10.3899/jrheum.131250

3. Coates LC, Mumtaz A, Helliwell PS, et al. Development of a disease severity and responder index for psoriatic arthritis (PsA)--report of the OMERACT 10 PsA special interest group. The Journal of rheumatology 2011;38(7):1496-501. doi: 10.3899/jrheum.110278

4. Acosta Felquer ML, Ferreyra Garrott L, Marin J, et al. Remission criteria and activity indices in psoriatic arthritis. Clinical rheumatology 2014;33(9):1323-30. doi: 10.1007/s10067-014-2626-y

5. Marin J, Acosta Felquer ML, Ferreyra Garrot L, et al. Patients with Psoriatic Arthritis Fulfilling the Minimal Disease Activity Criteria Do Not Have Swollen and Tender Joints, but Have Active Skin. The Journal of rheumatology 2016;43(5):907-10. doi: 10.3899/ jrheum.151101
6. Schoels M, Aletaha D, Funovits J, et al. Application of the DAREA/DAPSA score for assessment of disease activity in psoriatic arthritis. Annals of the rheumatic diseases 2010;69(8):1441-7. doi: 10.1136/ ard.2009.122259

7. Aletaha D, Alasti F, Smolen JS. Disease activity states of the DAPSA, a psoriatic arthritis specific instrument, are valid against functional status and structural progression. Annals of the rheumatic diseases 2016 doi: 10.1136/annrheumdis-2016-209511

8. Coates LC, Fransen J, Helliwell PS. Defining minimal disease activity in psoriatic arthritis: a proposed objective target for treatment. Annals of the rheumatic diseases 2010;69(1):48-53. doi: 10.1136/ard.2008.102053

9. Coates LC, Helliwell PS. Validation of minimal disease activity criteria for psoriatic arthritis using interventional trial data. Arthritis care \& research 2010;62(7):965-9. doi: 10.1002/acr.20155

10. Coates LC, Moverley AR, McParland L, et al. Effect of tight control of inflammation in early psoriatic arthritis (TICOPA): a UK multicentre, open-label, randomised controlled trial. Lancet 2015 doi: 10.1016/S01406736(15)00347-5

11. Kavanaugh A, Fransen J. Defining remission in psoriatic arthritis. Clinical and experimental rheumatology 2006;24(6 Suppl 43):S083-7. 